\title{
Endosome and INPP4B
}

Editorial

\section{Chen Li Chew, Ming Chen and Pier Paolo Pandolfi}

Phosphoinositide 3-kinase (PI3K) and AKT define a signaling network that regulates important biological processes such as cell cycle, survival, metabolism and motility, all of which are disrupted in cancer (Figure 1) [1]. Hyperactivation of PI3K leads to increased production of phosphoinositide (PI) species such as $\mathrm{PI}(3,4,5) \mathrm{P}_{3}$ and $\mathrm{PI}(3,4) \mathrm{P}_{2}$, thereby increasing membrane recruitment and activation of AKT, a key mediator of the oncogenic effects of enhanced PI3K signaling (Figure 1) [1]. Lipid phosphatases, like PTEN, control the levels of PI $(3,4,5)$ $\mathrm{P}_{3}$ to antagonize oncogenic PI3K/AKT signaling. INPP4B (inositol polyphosphate 4-phosphatase type II) has recently emerged as an important player in the regulation of PI3K-AKT signaling. INPP4B was initially identified as a potential tumor suppressor in a shRNA-mediated genetic screen performed in HMEC cells, where knockdown of INPP4B resulted in anchorage independent growth [2]. INPP4B preferentially hydrolyzes $\mathrm{PI}(3,4) \mathrm{P}_{2}$ to $\mathrm{PI}(3) \mathrm{P}$ [3] and, because the direct interaction of $\operatorname{PI}(3,4) \mathrm{P}_{2}$ with the pleckstrin homology domain of AKT is required for membrane recruitment and complete activation of AKT [1], INPP4B, like PTEN, should act as a tumor suppressor. The tumor suppressive role of INPP4B has been subsequently confirmed in two independent studies in vitro, where knockdown of INPP $4 B$ in basal-like breast cancer cell lines was found to increase cell proliferation, anchorage-independent growth and migration $[3,4]$.

Since the tumor suppressive role of INPP4B in vivo had not been addressed previously, we sought to further investigate 1) the tumor suppressive function of INPP4B both in vivo and in vitro with knockout mouse models, and 2) if Inpp $4 b$ loss cooperates with Pten heterozygosity in tumor progression. Our in vivo study showed that INPP4B is not solely epistatic to PTEN, since crossing Inpp $4 b^{-/-}$mice with $\mathrm{Pten}^{+/-}$mice did not accelerate the entire tumor spectrum of $\mathrm{Pten}^{+/-}$mice. Critically, we observed a specific acceleration of thyroid adenomas to metastatic follicular thyroid cancer (FTC), which resulted in the early mortality that occurred in the Pten ${ }^{+-} I n p p 4 b^{-1}$ mice [5]. Further studies using conditional Pten and Inpp $4 b$ knockout mice will be needed to determine the potential cooperative effect between PTEN and INPP4B in controlling tumorigenesis and metastasis in other tissues.

The cooperative roles of PTEN and INPP4B in suppressing tumor growth and metastatic FTC are evident in both our mouse model and in FTC patients where these two tumor suppressors are found concomitantly lost $[5,6]$. However, the specificity of such a phenotype suggests that while increased Akt activation plays a role in follicular-like thyroid tumorigenesis, it was insufficient in mediating progression and metastases, It also suggests that the tumor suppressive function of INPP4B was therefore extended beyond its role in suppressing the overall level of PI3K-AKT pathway activation. In this respect, a striking difference between PTEN and INPP4B emerged from our cellular fractionation experiments in their differential localization at the early endosome where INPP4B, but not PTEN, could regulate AKT2 signaling in a localized and specialized fashion [4].

Three highly homologous isoforms of AKT have been identified [1], however, despite their structural similarities, there is increasing evidence that these AKT isoforms are not functionally equivalent, rather, they promote distinct signaling outputs. Of note, AKT1 suppresses, while AKT2 promotes invasion, migration and epithelial-mesenchymal transition [1]. However, the mechanisms underlying specific AKT isoform activation and the identity of isoform specific substrates remain poorly understood. Through cellular fractionation experiments, we have provided evidence for the selective

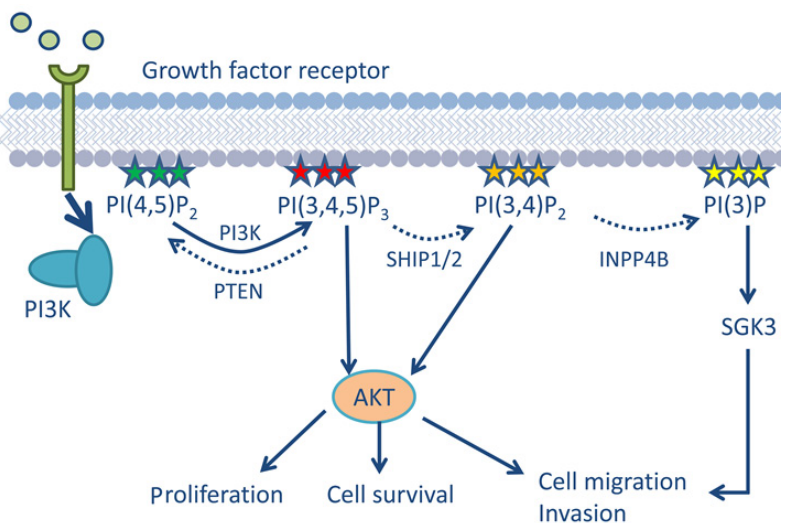

Figure 1: Regulation of PI3K/AKT signaling through phosphatases. Growth factor signaling results in the activation of $\mathrm{PI} 3 \mathrm{~K}$, which produces $\mathrm{PI}(3,4,5) \mathrm{P}_{3}$. $\mathrm{PI}(3,4,5) \mathrm{P}_{3}$ can subsequently be dephosphorylated by SHIP $1 / 2$ to $\operatorname{PI}(3,4)$ $\mathrm{P}_{2}$. Both $\mathrm{PI}(3,4,5) \mathrm{P}_{3}$ and $\mathrm{PI}(3,4) \mathrm{P}_{2}$ can activate $\mathrm{AKT}$, leading to cell proliferation, survival, migration and invasion. PTEN and INPP4B dephosphorylate $\mathrm{PI}(3,4,5) \mathrm{P}_{3}$ and $\mathrm{PI}(3,4) \mathrm{P}_{2}$ respectively, acting as tumor suppressors by antagonizing AKT signaling. Under certain circumstances, INPP4B can act as an oncogene PI(3)P, the product of INPP4B action, can activate SGK3, which activates pathways in proliferation, cell survival, migration and invasion. Figure adapted from Gewinner $C$ et al. Cancer Cell. 2009. 
regulation of AKT2 by INPP4B through its localization at early endocytic membranes.

Interestingly, PTEN has also recently been reported to be present on PI(3)P vesicles [7]. Although the exact identity and the cell type specificity of these vesicles remains to be determined, they could represent other endocytic signaling intermediates. The presence of tumor suppressive phosphatases on these membranes underscores the importance of regulating endocytic signaling intermediate, and provides an important link between endocytosis and cancer.

Surprisingly, recent studies have also indicated the oncogenic function of INPP4B in leukemia, breast, and colon cancer. This could be largely attributed to the product of INPP4B phosphatase activity (PI(3)P), which can drive AKT-independent and SGK3-mediated tumorigenesis [8], [Blood PMID: 25736236, Oncogene PMID: 26411369]. Similar to TGF $\beta$ and Notch signaling pathways, the dual activity exhibited by INPP4B in cancer regulation highlight the complexity of lipid signaling and endocytosis in cancer. The paradoxical nature of INPP4B's function could also potentially be attributed to differences in expression and localization of INPP4B isoforms in different tissues, and it would not be surprising if selective misexpression or downregulation of certain isoforms played a role in cancer progression.

Lastly, INPP4B did not appear to be deleted or mutated in human thyroid cancer, but rather, downregulated by gene methylation [5]. The use of epigenetic modifiers to upregulate INPP4B expression or therapeutic targeting of AKT2 may therefore present an effective strategy for the treatment of FTC. However, given the oncogenic role of INPP4B in certain cancers, these novel therapeutic treatments would need to be tailored to specific cancer types and subtypes, a key concept in developing precision medicine for cancer. Furthermore, our findings also suggest that activation of signal transduction pathways associated with endocytic trafficking is critical for tumor cell migration. As a consequence, selective targeting endocytic trafficking and signaling could potentially allow for the development of novel cancer therapies to prevent metastasis.
Pier Paolo Pandolfi: Cancer Research Institute, Beth Israel Deaconess Cancer Center, Department of Medicine and Pathology, Beth Israel Deaconess Medical Center, Harvard Medical School, Boston, MA, USA

Correspondence to: Pier Paolo Pandolfi, email ppandolf@ bidmc.harvard.edu

Keywords: cancer, metastasis, genetics, INPP4B, endosome, PI3K/AKT, thyroid

Received: November 19, 2015

Published: December 18, 2015

\section{REFERENCES}

1. Toker A, et al. Adv Biol Regul. 2014; 55: 28-38.

2. Westbrook TF, et al. Cell. 2005; 121: 837-848.

3. Gewinner C, et al. Cancer Cell. 2009; 16: 115-125.

4. Fedele CG, et al. Proc. Natl. Acad. Sci. U.S.A. 2010; 107: 22231-22236.

5. Chew CL, et al. Cancer Discov. 2015; 5: 740-751.

6. Kofuji S, et al. Cancer Discov. 2015; 5: 730-739.

7. Naguib A, et al. Mol Cell. 2015; 58: 255-268.

8. Gasser JA, et al. Mol Cell. 2014; 56: 595-607. 